\begin{tabular}{|c|c|c|c|}
\hline & & $15-23$ & Hannover 1995 \\
\hline
\end{tabular}

\title{
Ein marines Eem-Vorkommen im Binnenland Vorpommerns (Ton-Tagebau Grimmen, westlich Greifswald)
}

\author{
Gerhard STEINICH*)
}

Pleistocene, Eem-deposit, Stratigraphie, Environment, marine Deposits, Vorpommern, Greifswald

\begin{abstract}
Kurzfassung: Im Tagebau Grimmen (westlich Greifswald) ist marines Eem in transgressiver Überlagerung auf Saale-Geschiebemergel erschlossen. Die Kiese und Sande lassen sich als Vorstrand-Riff-Rinnen-Sedimente interpretieren. Da das Vorkommen als bodenständig gelten muß, wird eine weit in Talsysteme und Geländedepressionen eingreifende Eem-Überflutung für Vorpommern wahrscheinlich.

Die Deformation der Schichtenfolge, einschließlich der des Lias und Eozäns, ist älter als zwei (?drei) diskordante Weichsel-Geschiebemergel. Damit entfällt ein wesentliches Argument für die Existenz einer spätweichselzeitlichen Stauchendmoräne (Velgaster Staffel) in Vorpommern.
\end{abstract}

\section{[A marine Eem-deposit in Vorpommern - inland (clay open-pit mine of Grimmen; westerly of Greifswald)]}

\begin{abstract}
In the open-pit mine of Grimmen/westerly of Greifswald is placed a Eem-Interglacial onlap-sequence upon Warthe-moraine. The gravel and sand accumulations should be autochthonous. The Eem-Sea gave rize to a deep bay intruding on the land of Vorpommern.

The deformation of the sediments, including Lias and Eozän, should be older than the two (?or three) Weichsel-morains. The postulated push moraine of Velgaster Staffel does not exist in the Grimmen area.
\end{abstract}

\section{Einleitung}

Im Ost- und Südabschnitt des Tagebaues Grimmen (s. Abb. 1) bedeckt präweichselzeitliches Pleistozän den Lias-Ton. Es setzt sich aus einem basalen Geschiebemergel und mehreren Metern marine Fossilien führenden Kiesen und Sanden zusammen. In der deformierten und verstellten, aber insgesamt intakten Schichtenfolge gehören die Kiese und Sande der Eem-Warmzeit, der liegende Geschiebemergel der Saale-Kaltzeit an.

Die offenbar bodenständigen Sedimente und das erschlossene Profil führen zu veränderten Vorstellun-

\footnotetext{
*) Anschrift des Verfassers: Prof. Dr. G. SteInich, Am St.-Georgsfeld 36, 17489 Greifswald
}

gen über die Reichweite der Eem-Transgression in Vorpommern und über das Alter und die Dynamik des Deformationsprozesses der Schichtenfolge.

\section{Stratigraphie und lithologische Interpretation}

Unter zwei (?drei) Geschiebemergeldecken, einem mehrere Meter mächtigen Ablationsschutt und geringmächtigem Schluff (s. Abb. 2, Einheiten 10 bis 7) folgt diskordant ein ca. $24 \mathrm{~m}$ mächtiges älteres Pleistozän. Überwiegend liegt es dem Lias, stellenweise auch dem Eozän auf. Steil gestellt, zum Teil überkippt, ist die in sechs Einheiten gliederbare Schichtenfolge weitgehend intakt (s. Abb. 2, Einheiten 1 bis 6) und die Sedimentgefüge lassen eine geopedale Orientierung zu.

Einheit 1: Mit flachwelliger Basis setzt dem LiasTon ein etwa $14 \mathrm{~m}$ mächtiger, prismatisch zerfallender Geschiebemergel auf. Überwiegend ist er rotbraun, an seiner Dachfläche partieweise grün verfärbt. Geschiebearm, führt seine Matrix aufgearbeiteten Lias. Feinkiesanalysen ( 4 bis $10 \mathrm{~mm}$ ) ergeben ein baltisches Geschiebespektrum. Eine Grobkieszählung (unterer Teil des oberen Drittels) unterstreicht das (TGZ-Werte: $\lambda 16,69, \gamma 58,53$ ). Im Dachbereich zeigt sich eine Abreicherung von Kalken und eine Anreicherung von nordischem Kristallin und Feuerstein.

Einheit 2: Bis $27 \mathrm{~cm}$ mächtiger, feinsandiger, fein laminierter, olivbrauner bis rotbrauner Schluff. An seiner Basis enthält er vom Liegenden ausgehende Geschiebemergelschlieren, die bis auf Millimeter ausdünnen. Er führt Moosreste, reichlich Makrosporen von Selaginella und Ephippien von Daphnia. Eine Erosionsdiskordanz, die auch den liegenden Geschiebemergel erreicht, kappt ihn. In Form von Scheibengeröllen tritt dieser Schluff in den Einheiten 3 bis 5 auf. 


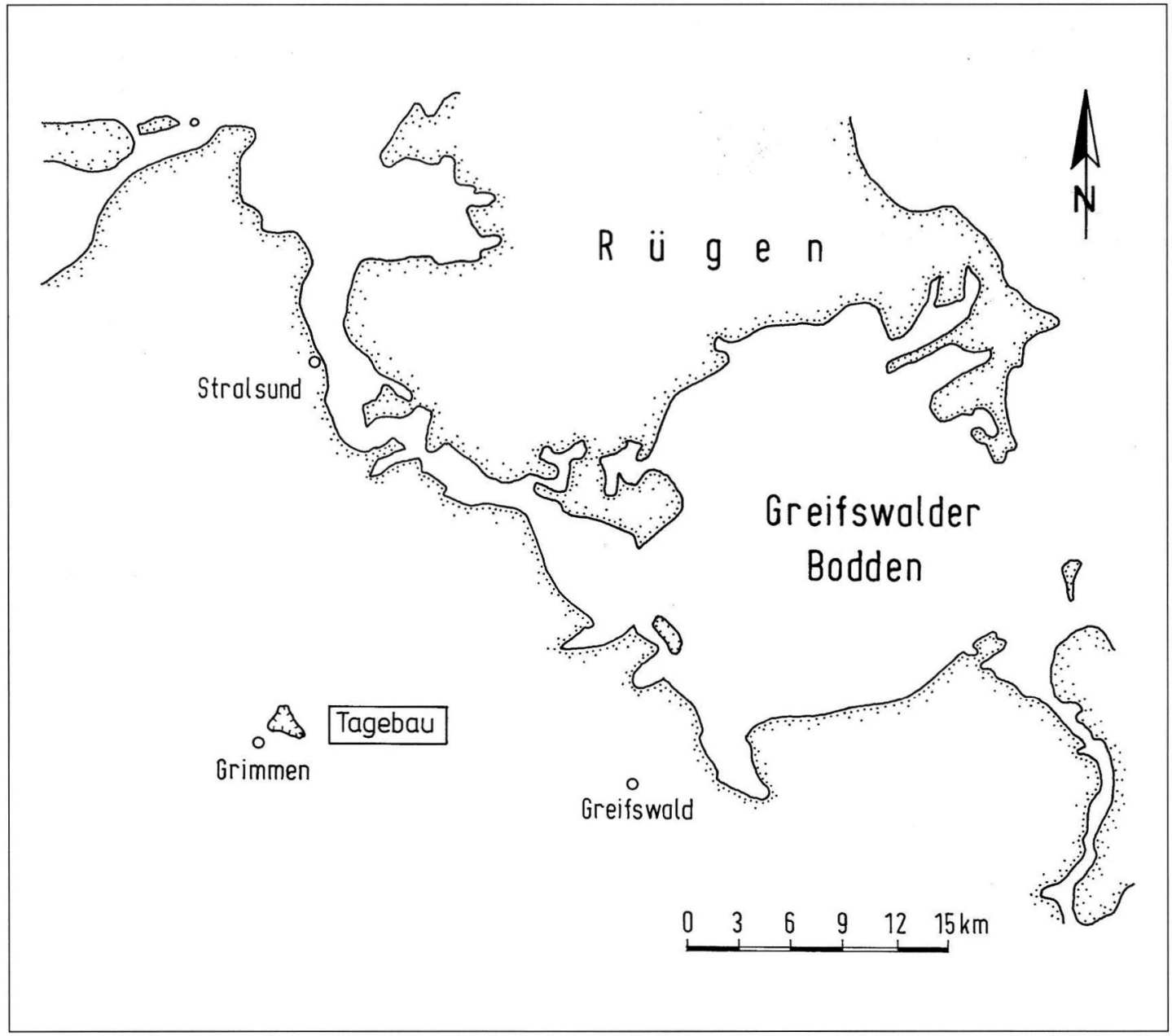

Abb. 1: Lageskizze des Tagebaues Grimmen

Fig. 1: Location of the opencast mine at Grimmen

Einheit 3: Kies mit Molluskenschalenbruch. Der gut gerundete Kies entstammt dem liegenden Geschiebemergel und Einheit 2. Sowohl Grobkieszählungen als auch Feinkiesanalysen entsprechen den Werten der Einheit 1 (TGZ: $\lambda 16,67, \gamma$ 58,55). Unterstrichen wird das durch Geschiebemergelbrocken aus dem Liegenden. Die Mächtigkeit reduziert sich von $1,0 \mathrm{~m}$ im Westteil (s. Abb. 2, Profil 1) auf $0,1 \mathrm{~m}$ ostwärts (s. Abb. 2, Profil 3). Das Artenspektrum des Schalenbruchs entspricht dem der Einheiten 4 bis 5.1 (siehe Einheit 5), er ist scharfkantig, z. T. lassen sich die Bruchstücke wieder zusammensetzen.

Einheit 4: Ohne Veränderung des Materialbestandes gegenüber Einheit 3 fällt Einheit 4 in Profil 1 durch eine gradierte Kies/Sand-Schichtung auf. 30 Lagen, zwischen $3 \mathrm{~cm}$ und $8 \mathrm{~cm}$ mächtig, lassen vom Liegenden zum Hangenden eine kontinuierliche Korngrößenabnahme und eine prozentuale Abnahme des Kiesanteils erkennen. Die obersten Lagen bestehen basal nur noch aus Mittel- bis Grobsand. Darin vorhandene Fossilreste sind sehr stark zertrümmert. Meist handelt es sich um Bruchstücke von dickschaligen Cardien und Venerupis. Scheibengerölle zeigen nordwärts gerichtete Imbrikation. Eine Erosionsdiskordanz begrenzt die Einheit gegen das Hangende.

Im Profil 2 tritt an die Stelle der gradierten Kies/Sand-Schichtung ein parallel- und schräggeschichteter Feinsand mit einzelnen Grobsandlaminen, Kreide-Feinkies- und Kohleanreicherungen, im höheren Abschnitt mit Erosionskolken, Strömungsrippelhorizonten und keilförmig kletternden Rippeln mit erosivem Kopf. Der Feinsand führt durchweg etwas Glaukonit. An Fossilien kommen darin neben vereinzelten Venerupis senescens (COCC.), Cerastoderma edulis (L), Mytilus edulis (L.), Turritella sp. folgende quartäre Formaminiferen vor: 


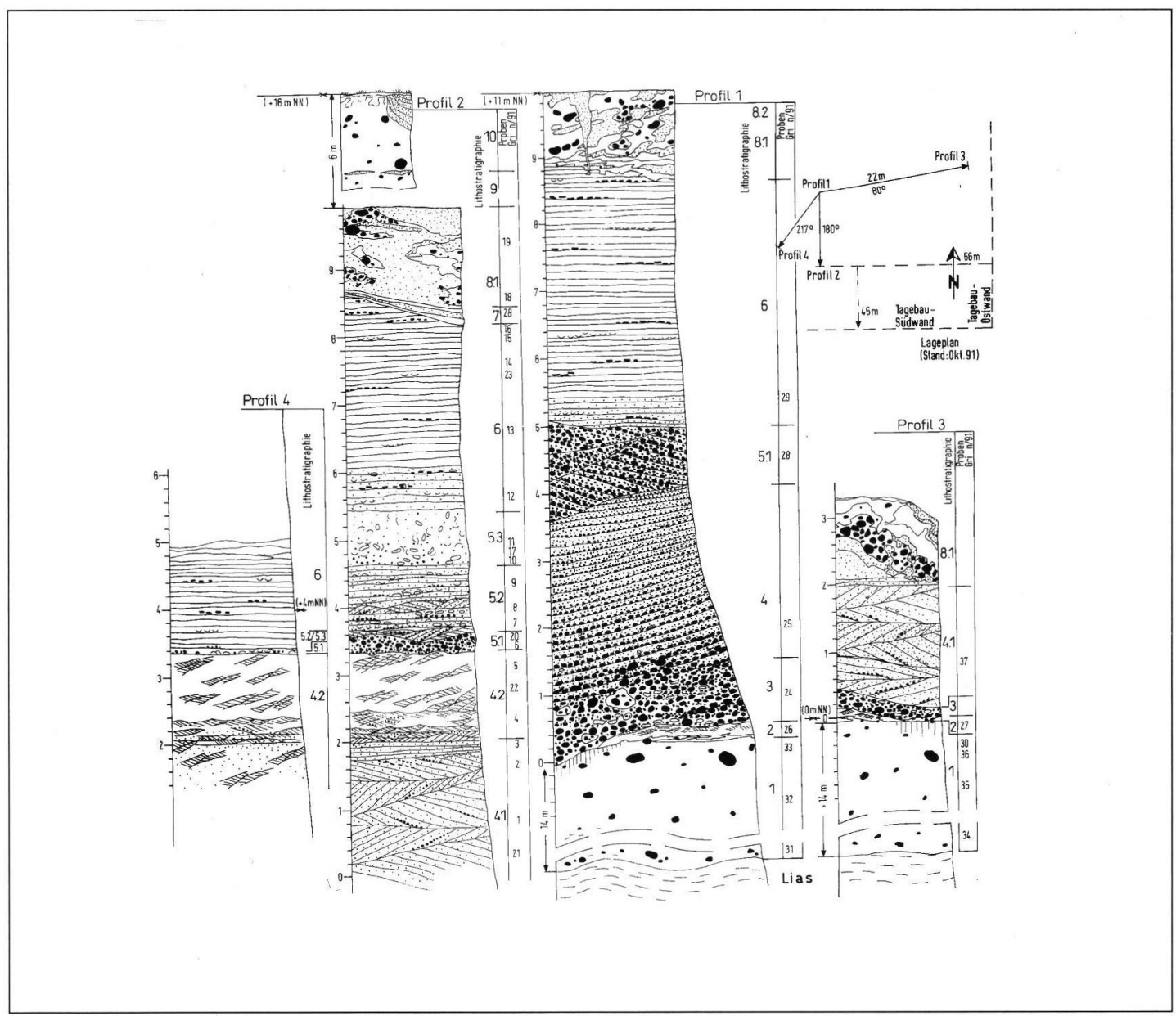

Abb. 2: Lithostratigraphie des Eem-Profils im Tagebau Grimmen.

Einheit 1: Rotbrauner Saale-Geschiebemergel, an der Oberfläche grün verfärbt (senkrechte Schraffen);

Einheit 2: Laminierter Schluff;

Einheit 3: Transgressionskies mit Erosionsdiskordanz an der Basis;

Einheit 4: Rhythmisch gradierter Sturmhochwasserkies;

Einheit 5.1: Kieswall mit Erosionsdiskordanz an der Basis und im Hangenden in Profil 1;

Einheit 5.2: Rippelgeschichteter Feinsand mit Scheibengeröllen der Einheit 2, Kieslagen und Schillanreicherungen;

Einheit 5.3: Schichtungsloser Feinsand mit chaotisch gelagertem Bivalvenbruch und Scheibengeröllen der Einheit 2;

Einheit 6: Parallelgeschichteter Feinsand, Kohleanreicherungen und Schillflecken;

Einheit 7: Laminierter Schluff;

Einheit 8.1: Ablationsschutt;

Einheit 8.2: Eiskeilpseudomorphose;

Einheit 9: Grauer, tonreicher Geschiebemergel;

Einheit 10: Brauner Geschiebemergel (?zwei) mit geringmächtigem Feinsand an der Basis, Sandmulden an der Ober fläche.

Fig. 2: Stratigraphy and sedimentary structures of the marine Eemian deposit in the opencast mine at Grimmen.

Unit 1: Red-brown Saale(Warthe)-till, greenish on the top (vertical hatching);

Unit 2: Laminated silt;

Unit 3: Transgressing gravel with erosional contact on the base;

Unit 4: Gradet gravel:

Unit 5.1: Gravel mound, erosional contact on the base and in top of the section 1;

Unit 5.2: Ripple-marked fine sand with flat-pebbles from Unit 2, gravel-levels and fossil debris;

Unit 5.3: Non-bedded fine sand with chaotical fossil debris and flat-pebbles from Unit 2

Unit 6: Uniform bedded fine sand with fragments of coal and shells;

Unit 7: Laminated silt;

Unit 8.1: Diamikton;

Unit 8.2: Sand wedge as unambiquous periglacial feature;

Unit 9: Grey clayey till;

Unit 10: Brown till (?two) with sand-level on the base, sand-filled trough on the top 


\section{Bulimina marginata}

D'ORBIGNy 1826

Cibicides sp.a

Cibicides sp.b

Dentalina sp.a

Dentalina sp.b

Globobulimina sp.

Globulina inaequalis

Reuss 1850

Elphidium albium-

bilicatum

(WeIss 1954)

Elphidium cuvillieri

LEVY 1966

Elphidium excavatum

var.

clavatum CusHMAN 1930

Elphidium gunteri

Cole 1931

Elphidium macellum

(FiCHTEL \& MOLL 1798)

Elphidium margaritaceum

CuSHMAN 1930

Islandiella sp.

Umgelagerte kretazische und tertiäre Foraminiferen bleiben selten.

Einheit 5: In Profil 2 lassen sich drei Untereinheiten unterscheiden:

5.1: 0,2 m mächtiger, schräggeschichteter Kieswall. Maximaldurchmesser der durchweg gut gerundeten Gerölle $10 \mathrm{~cm}$. Während westwärts (Profil 4) dieser Kieswall durch einen Bruchschill mit einzelnen Geröllen ersetzt wird, schwillt er nordwärts (Profil 1) auf 1,4 m Mächtigkeit an. Gegen das Hangende wird er von einer Erosionsdiskordanz geschnitten. Das Kies-Material entspricht dem Geschiebespektrum des liegenden Geschiebemergels.

5.2: Feinlaminierter, rippelgeschichteter Feinsand mit Kies- und Schillanreicherungen sowie Scheibengeröllen der Einheit 2. Muschelklappen stehen z. T. senkrecht zur Schichtung, oft liegen sie gewölbt unten. Es dominieren Spisula subtruncata (DA CosTA), relativ kleine Cardien und Gastropoden. Vereinzelt kommen Mytilusreste vor.

5.3: Schichtungsloser Feinsand mit rotbraunen Schluffgeröllen (Einheit 2) und reichlich Muschelschalenbruch. Die Schalenbruchstücke liegen meist gewölbt unten im chaotischen Durcheinander. 5.2 und 5.3 fehlen im Profil 1.
Makrofossilinhalt der Einheit 3 bis 5 :

Hydrobia ulvae (PENN.)

Bittium reticulatum (DA COSTA)

Hinia reticulata (L.)

Littorina littorea (L.)

Cerastoderma edulis (L.)

Mytilus edulis (L.)

Venerupis senescens (COcc.)

Spisula subtruncata (DA CostA)

(?) Mya sp.

Balanus sp.

Wesentliche Unterschiede in der Fossilführung zwischen den Einheiten 3 bis 5 bestehen nicht. Der Häufigkeit nach dominieren überall Cerastoderma, Venerupis, Bittium und Hinia. Nahezu $50 \%$ der Schalenbruchstücke sind Venerupis zuzuordnen. Die Bivalvenreste sind dickschalig und stammen von kräftig skulptierten Exemplaren (rekonstruierbare Schalengröße von Cerastoderma: $4-5 \mathrm{~cm}$ ).

Einheit 6: Maximal $4 \mathrm{~m}$ mächtiger Feinsand. Eine Laminierung setzt sich gegen das Hangende allmählich durch (Profil 2). Er enthält Kohleanreicherungen, selten Torf und Holzgerölle (darunter Larix), vereinzelt Gastropoden (Hinia reticulata, Bittium reticulatum), Spisula subtruncata und Cerastoderma. Die Muschelreste sind z. T. zu Schillflecken konzentriert, vereinzelt stehen Klappen hochkant in der Schichtung.

Ablationsschutt (Einheit 8) schneidet die ungestörten Profile im Hangenden. In Profil 2 bedeckt diskordant ein Schluff (Einheit 7) Einheit 6. Er enthält Moosreste und Oogonien von Nitella. Der Ablationsschutt wird von jüngeren Eiskeilpseudomorphosen durchschlagen (vertikale Länge ca. $3 \mathrm{~m}$. Öffnungsweite $0,4 \mathrm{~m}$, sterile Feinsandfüllung).

Zwei (?drei) zueinander konkordante Geschiebemergel schneiden die Schichtenfolge nach oben ab (Einheiten 9 und 10). Ein unterer (Einheit 9) ist grau, wird etwa $2 \mathrm{~m}$ mächtig, ist sehr geschiebearm und enthält, infolge der diskordanten Auflage auf den Lias, reichlich aufgearbeiteten Lias-Ton. Der obere (?zwei) ist weitgehend braun verwittert, partieweise entkalkt und erreicht 4,5 m Mächtigkeit.

Im oberen und in den unteren Geschiebemergel hineinreichend treten mehrfach Sandmulden auf. Sie enthalten Fließgeschiebemergeldecken, sterile Sande und Schluffe.

Marine Fossilien und Sedimente der Einheiten 3 bis 6 sind einander isochron, erstere befinden sich nicht umgelagert in jüngeren Bildungen, auch wenn zumindest die Kiese als ehemaliger Lebensraum nicht in Betracht kommen. Die Zeitgleichheit geht vor allem aus dem Erhaltungszustand des Fossilmaterials, aber auch aus dessen Orientierung im Sediment hervor. Die meist vollständigen Gastropodengehäuse 
wurden kaum abgerollt. Littorina littorea besitzt noch Farbzeichnung. Die Muschelklappen sind zwar weitgehend zertrümmert, doch die Bruchflächen haben scharfe Kanten. Bei der kleinen Spisula subtruncta ist das empfindliche Schloß gut erhalten. An einigen Wirbelstücken der Venerupis senescens sitzen noch Reste des Ligaments und an einzelnen Klappenbruchstücken sind auch Reste des Periostrakums vorhanden. Die Häufigkeit der quartären Foraminiferen in den Feinsandabschnitten des Profils ist bemerkenswert und spricht gegen Transportauslese.

Danach sind die Kiese und Sande für die darin vorhandenen Fossilien lebensraumnaher Einbettungsort und mariner Entstehung. Lithologisch wird das durch bimodale Korngrößenverteilungen gestützt (s. Abb. 3), die für küstennahe Sedimentationsräume charakteristisch sind (SHEA 1974, TAIRA \& SCHOlLE 1979, Flemming 1988). Die Sedimentgefügekombination spricht dafür, daß der Kies/Sand-Profilabschnitt eine Vorstrand-Riff-Rinnen-Sedimentation dokumentiert. Die marine Sedimentation beginnt mit einem Transgressionskies (Einheit 3). Die rhythmisch gradierten Sedimente der Einheit 4 sind Sturmhochwassersedimente. Einheit 5.1 ist als Strandriff und Einheit 6 als Sediment des Vorstrandes zu interpretieren. Das chaotische Sediment mit den z. T. hochkant stehenden Muschelklappen der Einheiten 5.2 und 5.3 spricht für Resedimentationsprozesse. Während der untere Teil des Profils 1 vorran-

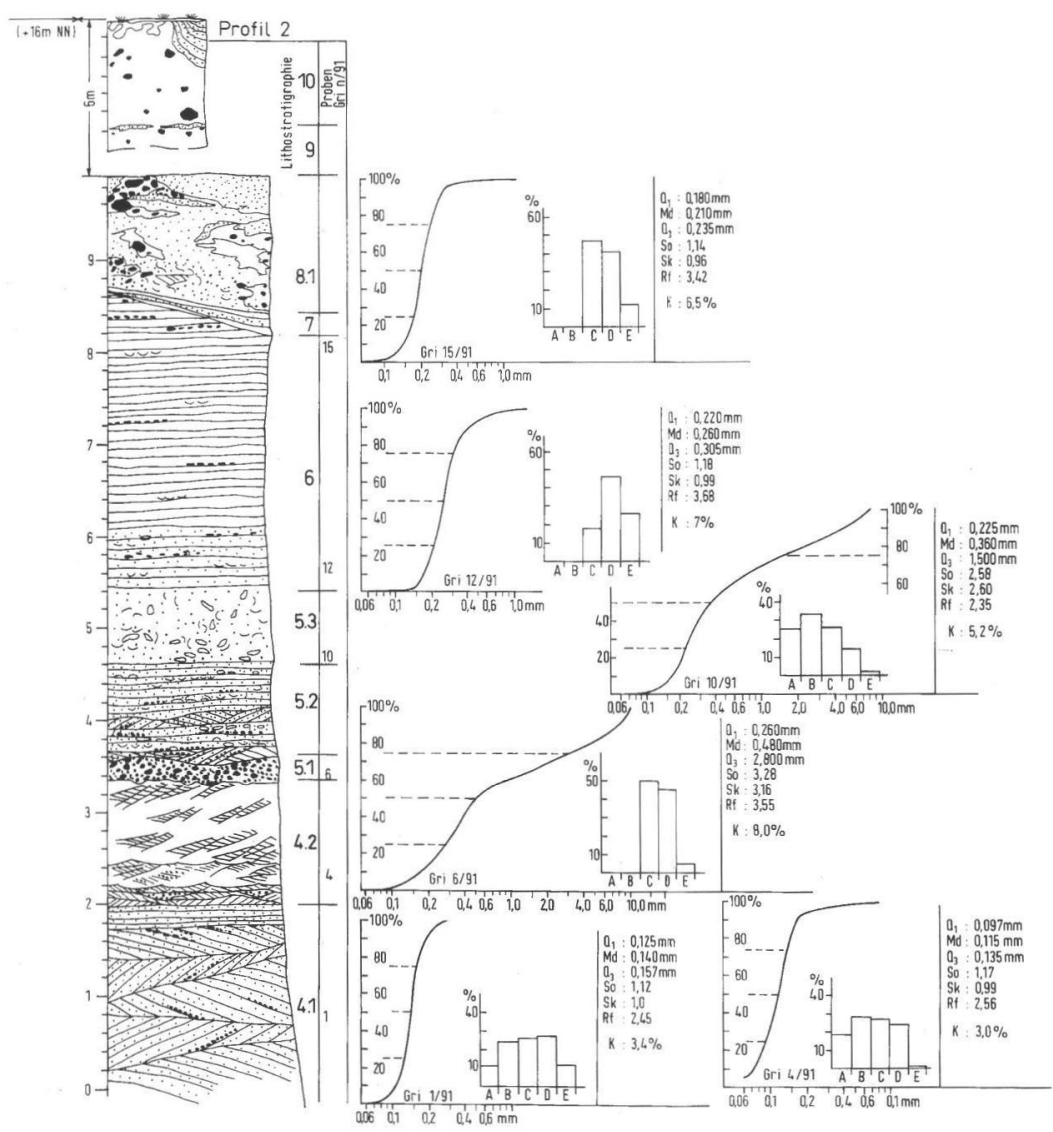

Abb. 3: Korngrößenverteilung und Rundungsfaktor einiger typischer Proben des Profils 2 von Grimmen. Q1, Md, Q3 = Quartil-Maße (Quadratlochsiebung, trocken, maschinell, 2 × 7,5 min); So = Sortierungskoeffizient, Sk = Schiefekoeffizient (nach Trask);

$\mathrm{Rf}=$ Rundungsfaktor (RUssel \& TAYLOR), $\mathrm{A}=$ angular, $\mathrm{B}=$ subangular, $\mathrm{C}=$ angerundet, $\mathrm{D}=$ gerundet, $\mathrm{E}=$ gut gerundet $(100$ Körner, visuell unter Binokular; Korngrößen 0,100/0,125 bis 0,160/0,200 mm, die Proben Gri 10/91 und Gri $15 / 91$ Korngrößen 0,200 bis 0,250 mm), Gri 12/91 Korngröße 0,250 bis 0,315 mm, K = Kalkgehalt der Fraktion < 0,063 mm (SCHEIBLER).

Fig. 3:Grainsize analysis and rounding several samples from section 2 in the opencast mine at Grimmen.

$\mathrm{Q} 1, \mathrm{Md}, \mathrm{Q} 3=$ moment measures; So = sorting; Sk = skewness; Rf = roundness (Russet \& TAYLOR), $\mathrm{A}=$ angular, $\mathrm{B}=$ subangular, $\mathrm{C}=$ subrounded, $\mathrm{D}=$ rounded, $\mathrm{E}=$ well rounded (100 grains, grainsize $0,100 / 0,125$ to $0,160 / 0,200 \mathrm{~mm}$, samples Gri 10/91 and Gri $15 / 91$ grainsize 0,200 to 0,250 ), Gri $12 / 91$ grainsize 0,250 to $0,315 \mathrm{~mm} ; \mathrm{K}=\mathrm{CaCO}_{3}$ content grainsize $<0,063 \mathrm{~mm}$ (SCHEIBLER). 
gig einen strandnahen Riffbereich dokumentiert, enthält Profil 2 Strandprielbildungen, mit den Auftauchanzeichen gekappter Rippeln und erheblichem äolischem Sandeintrag. Insgesamt liegt eine typische „onlap“-Sequenz vor und die retrogradierte Schichtfolge dokumentiert eine Transgression.

Nach dem Makro- und Mikrofossilinhalt ist der marine Profilabschnitt dem Eem und damit der liegende Geschiebemergel der Saale-Kaltzeit zuzuordnen. Nimmt man die für Saale-Moränen von MEYer (1983) als kennzeichnend angegebenen TGZ-Werte der Leitgeschiebe auch für Vorpommern als zutreffend an, läßt der Geschiebemergel sich als Warthe-zeitlich einstufen. Damit stimmen auch die empirischen Merkmale überein (Färbung, Geschiebehäufigkeit, Festigkeit). Das veränderte Geschiebespektrum in seinem Dach könnte eine festländische Verwitterungsphase andeuten, während die grüne Verfärbung jünger ist und vielleicht als Reduktionszone unter aquatischen Bedingungen entstand.

Der reliktisch dem Saale-Geschiebemergel aufliegende Schluff (Einheit 2) ist ein Süßwassersediment, das sich nach den Florenresten an die vorausgehende Kaltzeit anschließt (Spät-Saale).

Die Einheiten 7 bis 10 gehören dem Weichsel an, ohne daß zur Zeit genauere stratigraphische Aussagen möglich sind.

\section{Geologische Folgerungen}

Gewöhnlich werden die oberflächennahen Vorkommen von Lias, untergeordnet auch Kreide und Eozän, im Gebiet von Grimmen als glazigener Schollenschwarm aufgefaßt, dynamisch mit aktivem Weichsel-Inlandeis in Verbindung gebracht (GEHL 1965) und seit ElBert (1907, S. 181) als Beleg für den Verlauf einer Stauchendmoränenstaffel (Velgaster Staffel; Hesemann 1933, S. 72, Abb. 9) betrachtet. HeSEMANN zeichnete als erster im Gebiet von Grimmen ein nach Westen konvexes Stück einer Endmoräne. Spätere Autoren, mit Ausnahme von RichTER (1937), behielten das nicht nur bei, sondern zogen mit geschlossenem Bogen eine Stauchendmoräne höherer Ordnung durch Vorpommern (REINHARD \& SCHULTZ 1961, KLIEWE \& JAHNKe 1972). Dieser Auffassung entgegenstehende Folgerungen lassen die im gegenwärtig großräumigen Tagebau Grimmen erschlossenen geologischen Verbandsverhältnisse zu.

Der Saale-Geschiebemergel steht mit dem Grimmener Lias im primären Kontakt. Das geht aus dem in die Matrix reichlich aufgenommenen Lias, aus der Weiträumigkeit dieser Kontaktfläche im Tagebau und aus der gemeinsamen Deformation hervor. Auch sein Übergreifen über Eozän stützt das. Die Lias-Saale-Eem-Sedimente sind nicht als wurzellose
Scholle im jüngeren Pleistozän interpretierbar. Für eine Verbindung mit dem Anstehenden sprechen folgende Beobachtungen, auch wenn unter dem Lias z. T. pleistozäne Sande erbohrt sind (WILlmer 1960):

1. Aus den angeschnittenen Sanden und Schluffen des Domer tritt Sole aus, während die pleistozänen Sande, auch die des Eem, überall nur Süßwasser liefern. Trotz gemeinsamer Deformation blieben die pleistozänen Sande vom versalzenen Grundwasser des Domer isoliert. Die Mineralisation des Wassers im Domer geht auf abgelaugte Salze des Untergrundes (?Trias) zurück, und als Aufstiegswege kommen Bruchstörungen des mesozoischen und noch im Tertiär aktiven NE-mecklenburgischen Störungssystems in Betracht (WEGNER 1966, BRÜCKNER \& PETZKA 1967, BeUTLER 1978).

2. Der Lias der seit Richter (1933) bekannten Struktur Grimmen und auch der unmittelbar östlich benachbarten Struktur Reinkenhagen (PETZKA 1961) taucht umlaufend unter Kreide und unter Eozän ab. Die oberflächennahen Liasvorkommen mit dem aufliegenden Saale/Eem sind damit nicht aus umliegenden Gebieten herleitbar und müssen als bodenständig betrachtet werden. Eine Autochthonie des Lias von Grimmen hielten schon ERnST (1967, 1991), Wendland (1976) und Roselt (1984) für wahrscheinlich.

Tektonisch bildet die Kontaktfläche zwischen Lias und Saale-Geschiebemergel eine Aufwölbung ab. Die Schichtflächen, Faltenachsen und Kluftflächen lassen sich nicht einem einheitlichen System zuordnen. Roselt (1984) glaubt nach einer tektonischen Analyse des Lias (s. Abb. 4) einen mehraktigen Deformationsvorgang erkennen zu können, wobei die Ausbildung der Hauptbewegungsbahnen und der tektonische Hub der Sedimente älter sind als eine jüngere Deformation. Die letzte Druckbeanspruchung weist auf Dominanz einer nordöstlichen Hauptnormalspannungsrichtung hin ( $44^{\circ}$ : Klüfte, Scherflächen, Harnische). Ein östlicher Schub, wie er entsprechend der von ReINHARD und Schultz (1961) konstruierten Endmoräne zu fordern wäre, beherrscht das Bild nicht.

Zwei (?drei) jüngste, ungestört lagernde Geschiebemergel greifen diskordant über Lias, Eozän und das ältere Pleistozän hinweg und bedecken geschlossen das Gesamtgebiet. Hub und Deformation des Lias, des Eozän und des Saale/Eem waren vor Auflagerung des ältesten dieser Geschiebemergel schon vollzogen. Das belegt der diskordante Schluff der Einheit 7 (s. Abb. 2, Profil 2). Damit stehen die Liasvorkommen von Grimmen mit ihrer Saale/Eem-Auflage als Argument für eine spätweichselzeitliche Stauchmoräne nicht mehr zur Disposition. 

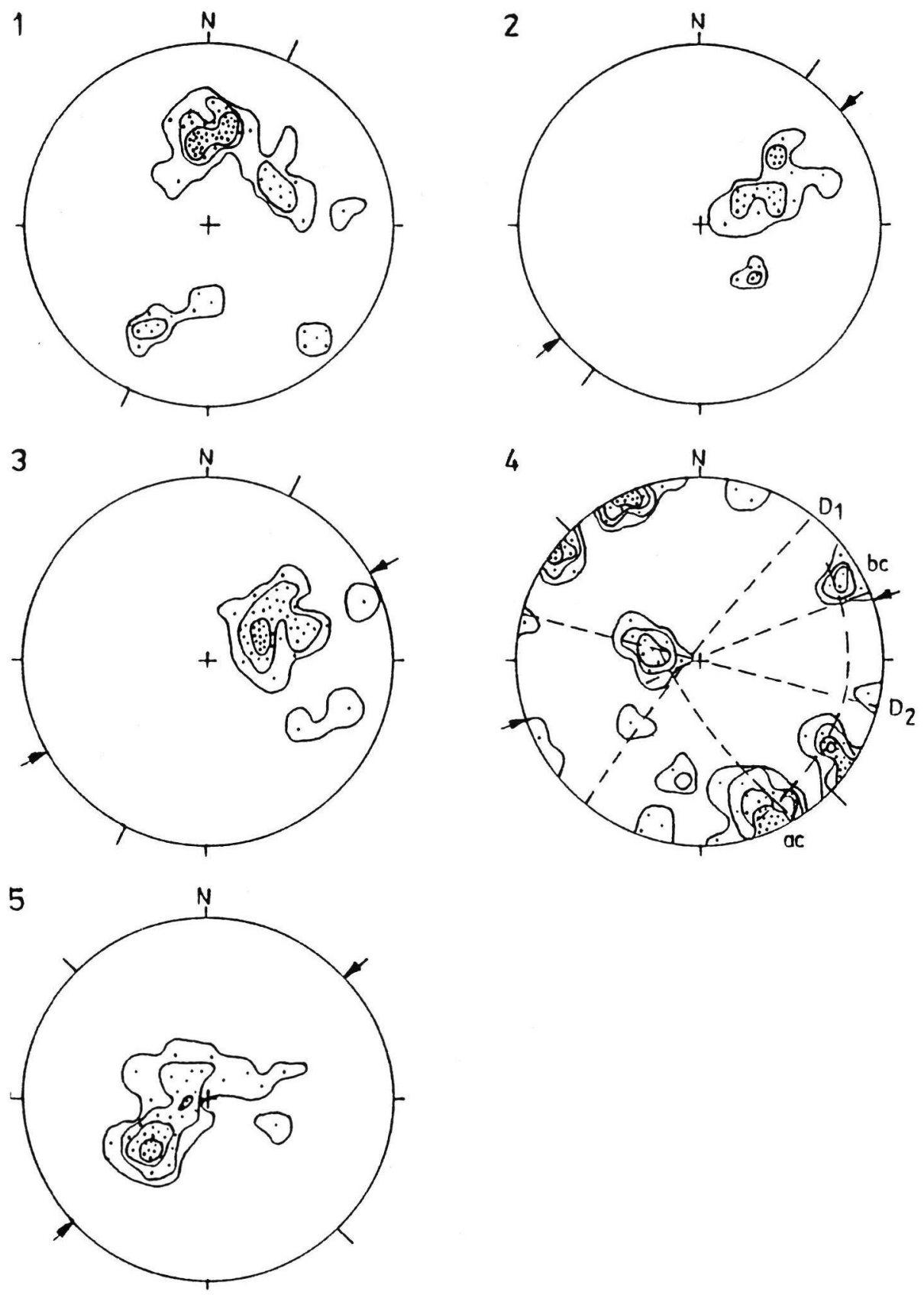

Abb. 4: Tektonische Meßwerte aus dem Grimmener Lias (Roselt 1984: Abb. 89-93, S. 56, umgezeichnet).

1: Klüfte im Ton des Lias $\epsilon$ (25 Werte: $1-2-4-4) ; 2$ : Störung Lias $\delta /$ Lias $\epsilon$ (15 Werte: $1-2-3-3)$; 3 : Schichtung des Lias $\delta$ (21 Werte: 1-2-4-4); 4: Klüfte im Lias $\delta$ (40 Werte: 1-2-3-4-5-5); 5: Störung Lias $\delta$ /Lias $\epsilon$ (30 Werte: 1-3-5-9-9).

Durchstoßpunkte der Flächennormalen (ScHmidTsches Netz, untere Lagenhalbkugel); D1, D2 = Großkreise der Diagonalklüfte; $\mathrm{ac}, \mathrm{bc}=$ Elemente des orthogonalen Kluftsystems; Pfeile = Druckrichtung.

Fig. 4: Tectonic structure of the Jurassic (Lias) clay in the opencast mine at Grimmen (ROSELT 1984).

1: Shear planes in the Lias $\boldsymbol{\epsilon}(\mathrm{n}=25)$; 2: Fault Lias $\delta /$ Lias $\boldsymbol{\epsilon}(\mathrm{n}=15)$; 3: Bedding Lias $\delta(n=21)$; 4: Shear ples in the Lias $\delta$ ( $n=40)$;

5: Fault Lias $\delta /$ Lias $\boldsymbol{\epsilon}(\mathrm{n}=30)$. Directional elements. 
Die an der Oberfläche vorhandenen, in Umriß und Größe unregelmäßigen Sandmulden sind keine Stauchungsergebnisse. Auch als Schmelzwassererosionsrinnen lassen sie sich nicht interpretieren. Dagegen sprechen die Sedimentgefüge und die Lagerung. Sie haben gegen die randlichen Geschiebemergel steile, z. T. senkrechte Begrenzungen, sind mit Fließgeschiebemergeldecken, die sich aus den Flanken entwickeln und gegen das Zentrum ausdünnen, auch mit Sanden und Schluff verfüllt. Die im unteren Teil stark muldenförmige und z. T. auch gestörte Schichtneigung nimmt gegen die Geländeoberfläche bis zur Horizontalen allmählich ab. Die Sandmulden sind als verfüllte Toteiswannen zu deuten.

Was als Argument für die Existenz einer Velgaster Stauchmoränenstaffel im Gebiet von Grimmen bleibt, sind einige Höhenrücken, die von Talrinnen begleitet werden. Schon STACH (1935, S. 5) stellte fest, daß von einer geschlossenen Endmoräne im Grimmener Gebiet keine Rede sein kann und höchstens morphologisch einige Höhenrücken mit einer Eisstillstandslage in Verbindung gebracht werden könnten. Bei großmaßstäbiger Betrachtung fügen die flachen Höhenrücken sich nicht zu einem Bogen zusammen, der als Endmoräne interpretiert werden kann. Sie lösen sich winklig auf, wie auch die Schmelzwasserrinnen ein winkliges System erkennen lassen, das keine Beziehungen zu einer Endmoräne hat. Eher ist an ein eisspaltenkontrolliertes Eiszerfallsrelief zu denken, eine Vorstellung, die schon v. BüLOw (1930) und später RICHTER (1937) vertraten.

Das oberflächennahe Liasvorkommen mit der Saale/Eem-Decke im Gebiet von Grimmen ist durch die präpleistozäne Liashochlage von Grimmen bedingt. Das seit dem Mesozoikum wirksame strukturelle Grundkonzept dieses Raumes lebte nicht nur im Tertiär neu auf (BrüCKNer \& PETZKa 1967, Beutler 1978), es scheint auch im Pleistozän wirksam gewesen zu sein. Dabei lassen sich Hub und Deformation der Sedimente nicht mit einer allgemeinen Druckwirkung eines vorstoßenden Gletschereises in Verbindung bringen. Die Beobachtungen sprechen eher für Aufstieg während einer Entlastungsphase.

Wenn nach obigen Feststellungen das Eem von Grimmen als bodenständig gelten muß, ist mit einer weit ins Binnenland Vorpommerns eingreifenden Eem-Überflutung zu rechnen. Wie in NordwestDeutschland sind dafür Talungen und Geländedepressionen prädestiniert. Unter Berücksichtigung des während des Eem erreichten maximalen Seespiegelstandes (DECHEND 1958, SINDOWSKI 1958, 1965) erscheint es möglich, daß das weitere Gebiet des mecklenburg-vorpommerschen Grenztales, zu dem der Grimmener Raum gerechnet werden kann, in diesem Sinne wirksam war. Letzteres würde bedeuten, daß die als spätweichselzeitlich angelegt geltende Niederung des Grenztales schon vor dem Eem existierte und später als Schmelzwasserrinnensystem nur wiederbenutzt wurde.

\section{Dank}

Für die Bestimmung von Fossilmaterial danke ich den Herren

E. Strauch, Münster (Spisula subtruncata [DA COSTA]),

P. FRENZEL, Greifswald (Foraminiferen, Ostracoden), C. SChirarend, Berlin (Larix),

für Geschiebezählungen

W.-A. Panzig, Greifswald und

U. STRAHL, Berlin.

\section{Schriftenverzeichnis}

Beutler, G. (1978): Der Einfluß der Möckow-Dargibeller Störungszone auf Sedimentationsprozesse im Mesozoikum. - Z. geol. Wiss., 6 (3): 319-328, Berlin (Akademie-Verlag).

BrüCKNER, W. \& PETzKA, M: (1969: Paläogeographie und Lagerungsverhältnisse von Alb und Oberkreide in NEMecklenburg (Raum Rügen-Usedom). - Ber. deutsch. Ges. geol. Wiss., A, 12 (5): 521-533, Berlin (AkademieVerlag).

BüLow, V. K. (1930): Landschaftliches und Erdgeschichtliches über die Gegend von Grimmen. - Unser Pommernland, Sonderh. Kreis Grimmen, Monatsber. f. d. Kulturleben d. Heimat: 264-269, Stettin (Fischer \& Schmidt).

DECHEND, W. (1959): Marines und brackisches Eem im Raum der Eems-Mündung. - Geol. Jb., 76: 175-190, Hannover.

ELBERT, I. (1907): Die Entwicklung des Bodenreliefs von Vorpommern und Rügen, sowie den angrenzenden Gebieten der Uckermark und Mecklenburgs während der letzten diluvialen Vereisung. - Jahresber. geogr. Ges. Greifswald, 10: 61-221, Greifswald.

ERNST, W. (1987): Lias-Tontagebau Grimmen. - Exkf. OttoJaekel-Symposium Greifswald, 17-31, Greifswald. Unveröff

- (1991): Lias im Tontagebau bei Grimmen (Vorpommern). - Fundgrube, 27 (4): 171-183, Berlin (Meteor).

Flemming, B. W. (1988): Process and pattern of sediment mixing in a microtidal coastal lagoon along the west coast of south Africa. - In: de BOER et al. (eds.): Tide-influenced Sedimentary Environments and Fazies: $275-$ 288, Dordrecht.

GEHL, O. (1965): Gesetzmäßigkeiten in der Verbreitung der Schollen im Jungpleistozän Mecklenburgs. - Geologie, 14 (5/6): 663-676, Berlin (Akademie-Verlag).

Hesemann, J. (1933): Zur Geschiebeführung und Geologie des Odergletschers. 1. Äußere, Rosenthaler und Velgaster Randlage. - Jb. preuß. geol. Landesanst., 53: 70-84, Berlin. 
KLIEWE, H. \& JaHNKE, W. (1972): Verlauf und System der Marginalzonen der letzten Vereisung auf dem Territorium der DDR. - Wiss. Z. Univ. Greifswald, Math.-naturw. R., 21 (1): 31-37, Greifswald.

MEYER, K.-D. (1983): Indicator pebbles and stone count methods. - In: EHLERS, J. (Hrsg.): Glacial Deposits in North-West Europe: 275-287, Rotterdam (Balkema).

PETZKA, M. (1961): Die Kartierungsbohrung Grimmen 13/60. - Ergebnisber., Arch. Geol. Erkdg. Nord. Schwerin. Unveröff.

ReINHARD, H. \& SCHUlz, H.-J. (1961): Zur Morphologie und Genese des Rycktal-Zungenbeckens. - Geogr. Berichte. 6 (1), 10-25, Berlin.

RICHTER, K. (1933): Gefüge und Zusammensetzung des norddeutschen Jungmoränengebietes. - Abh. geol.pal. Inst. Univ. Greifswald, 11: 1-63, Greifswald.

-- (1937): Die Eiszeit in Norddeutschland. - 179 S., Berlin (Borntraeger).

RoselT, K. (1984): Das tektonische Interngefüge der LiasScholle von Grimmen und der Tertiär-Schollen von Friedland im Vergleich zu ihrer strukturgeologischen Position im Quartär. - Dipl.-Arbeit Univ. Greifswald, 82 S.; Greifswald. Unveröff.

SHEA, J. H. (1974): Deficiencies of clastic particles of certain sizes. - J. Sediment. Petrol., 44, 985-1003, Tulsa.
SINDOWSKI, K.-H. (1958): Das Eem im Wattgebiet zwischen Norderney und Spiekeroog, Ostfriesland. - Geol. Jb., 76: 151-174, Hannover.

- (1965): Das Eem im ostfriesischen Küstengebiet. - Z. dt. geol. Ges., 115: 163-166, Hannover.

STACH, E. (1935): Erläuterungen zu Blatt Grimmen Nr. 512, Geologische Karte von Preussen und benachbarten deutschen Ländern: 1-12, Berlin.

TAIRA, A. \& SCHOLLE, P. A. (1979): Origin of bimodal sands in some modern environments. - J. Sedim. Petrol., 49: 777-786, Tulsa.

Wegner, J. (1966): Strukturbau und Tektonik im NE der DDR. - Geophys. Geol., 9: 44-56, Leipzig.

WendLAND, F. (1976): Zur Strukturentwicklung schwach dislozierter Salinarstrukturen in Nordostmecklenburg, Strukturen Grimmen und Reinkenhagen (Beiträge zur Mächtigkeitsanalyse von Salinaren). - Veröff. ZIPE d. Akad. d. Wiss. DDR, 33: 1-118, Potsdam.

Willmer, H. (1960): Geologische Erkundung und Vorratsberechnung des Tonvorkommens Grimmen. - Ergebnisbericht, Arch. Geol. Erkdg. Nord. Schwerin. Unveröff.

Manuskript eingegangen am 21. 12. 1993 